\title{
Riqueza e densidade de vocalizações de anuros (Amphibia) em uma área urbana de Corumbá, Mato Grosso do Sul, Brasil
}

\author{
Robson Waldemar Ávila ${ }^{1} \&$ Vanda Lúcia Ferreira ${ }^{2}$ \\ 1 Pós-graduação em Ecologia e Conservação, Universidade Federal de Mato Grosso do Sul. Caixa Postal 549, 79070-900 \\ Campo Grande, Mato Grosso do Sul, Brasil. E-mail: robsonavila@nin.ufms.br \\ 2 Laboratório de Zoologia, Departamento de Ciências do Ambiente, Universidade Federal de Mato Grosso do Sul. Avenida Rio \\ Branco 1270, Caixa Postal 252, 79301-970 Corumbá, Mato Grosso do Sul, Brasil.
}

\begin{abstract}
Richness of species and density of vocalization of anurans in an urban area of corumbá, mato grosso do sul, brazil. The richness and intensity of vocalization of anurans in urban areas of Corumbá, State of Mato Grosso do Sul, Brazil, were studied from February 2002 to January 2003 in a secondary forest and surroundings of a temporary pond. We used a transect with four stop sites on which the presence of species and the intensity of vocalization were recorded according to North American Amphibian Monitoring Population index. The turn when vocalization happened was also recorded. We registered 16 species of four families: Bufonidae (2), Hylidae (7), Microhylidae (1), and Leptodactylidae (6). The reproduction of these species was correlated to the rainy season, from December to March $\left(r^{2}=0,806, F(1,10)=41,530 p=0,002, n=12\right)$. The species that presented greater intensity of vocalization were Scinax nasicus (Cope, 1862) and Physalaemus albonotatus (Steindachner, 1864), with peaks during January. The species that presented the greater period of vocalization was Leptodactylus fuscus (Schneider, 1799), from September to February. The majority of species and individuals vocalized from 7:00 p.m. to 11:00 p.m.

KEYWORDS. Amphibian, Pantanal, temporal distribution, temporary pond.
\end{abstract}

RESUMO. A riqueza e intensidade de vocalizações de anuros em uma área urbana de Corumbá, Mato Grosso do Sul, Brasil, foram estudadas de fevereiro de 2002 a Janeiro de 2003 em uma poça temporária. Foi utilizado um transecto com quatro pontos de escuta onde foram consideradas as espécies visualizadas e a intensidade das respectivas vocalizações. Foram registradas 16 espécies de quatro famílias: Bufonidae (2), Hylidae (7), Microhylidae (1) e Leptodactylidae (6). $O$ período reprodutivo dessas espécies esteve correlacionado com a estação chuvosa, de dezembro a março $\left(r^{2}=0,806, F(1,10)=41,530 p=0,002, n=12\right)$. As espécies que apresentaram maior intensidade de vocalização foram Scinax nasicus (Cope, 1862) e Physalaemus albonotatus (Steindachner, 1864), com picos em janeiro. A espécie que apresentou maior período de vocalização foi Leptodactylus fuscus, de setembro a fevereiro. O maior número de espécies e de indivíduos vocalizando ocorreu de 19:00 às 23:00 $\mathrm{h}$.

PALAVRAS CHAVE. Anfíbios, distribuição temporal, Pantanal, poça temporária.

A atividade de vocalização dos anuros em determinado horário é condicionada por caracteres fisiológicos relacionados ao seu modo de vida. Devido à necessidade do meio aquático para sobrevivência das larvas, o acasalamento na maioria das espécies de anuros tende a ocorrer em épocas restritas do ano, condicionadas principalmente pelas chuvas e pela temperatura (CARdoso \& Martins 1987).

Recentemente, a conservação dos anfíbios tem recebido considerável atenção, sobretudo após as informações sobre a redução drástica de muitas populações (FerRIER 2002). Várias causas são apontadas para essa diminuição, dentre elas a destruição de hábitats (PAPP \& PAPP 2000, MAZEROlle 2001), intro- dução de espécies exóticas (SEEbaCher \& Alford 1999), tráfico ilegal (Summers 2002) e o desenvolvimento urbano (JANSEN et al. 2001). No entanto, algumas populações podem apresentar flutuações naturais, dificultando a percepção dos impactos antrópicos (Pechmann et al. 1991, Marsh 2001).

O desenvolvimento urbano degrada e fragmenta hábitats naturais, limitando a dispersão e alterando as condições climáticas locais, além de favorecer as espécies exóticas (KoENIG et al. 2002). Além disso, muitos animais podem ser mortos intencional ou acidentalmente, como por exemplo, atropelados (CARR \& FAHRIG 2001). Anfíbios são particularmente suscetíveis à urbanização devido à necessidade de água em seu ciclo vital

Revista Brasileira de Zoologia 21 (4): 887-892, dezembro 2004 
(JANSEN et al. 2001). Para tanto, fazem-se necessários programas de monitoramento a longo prazo (WILSON \& M ARET 2002) e conhecimento do comportamento e ecologia das populações de anuros (HodgKISON \& HeRo 2001).

O Município de Corumbá, localizado no Pantanal de Mato Grosso do Sul, à margem do rio Paraguai, vem passando por intenso processo de urbanização, com incentivo à industrialização e criação de um pólo minero-siderúrgico, podendo interferir drasticamente na populações de anuros da área urbana. Neste trabalho são apresentados dados sobre a riqueza e densidade de vocal izações de anuros naquela área urbana.

\section{MATERIAL E MÉTODOS}

\section{Área de coleta}

As coletas foram realizadas na mata da empresa Cimento Itaú (18058'48"S, 57039'18"W), localizada na área urbana de Corumbá, na divisa com o município de Ladário, Mato Grosso do Sul. A vegetação é secundária, com predominância de árvores das famílias Euphorbiaceae, Anacardiaceae e Leguminosae. As chuvas concentram-se de outubro a março (Fig. 1), formando uma lagoa temporária que desaparece no período de estiagem dando lugar às gramíneas. De acordo com a classificação de Köppen, o clima de Corumbá é do tipo Awa, ou seja, tropical de altitude, megatérmico, com inverno seco e verão chuvoso (SORIANO 1997).

\section{Metodologia}

O registro da riqueza e intensidade de vocalização das espécies de anuros foi realizado de fevereiro de 2002 a janeiro de 2003, e cada amostragem teve cinco dias consecutivos de observação, entre aproximadamente 17:00 h e 06:00 h. Um transecto com quatro pontos foi percorrido e o registro das espécies foi feito através de vizualização e vocal ização. A seqüência de registros por pontos foi realizada por sorteio sem reposição. Animais encontrados em habitações humanas foram considerados na riqueza total do local. Os registros foram realizados na fase crescente da lua, minimizando a interferência em potencial do padrão de atividade (ver DuelLman \& Trueb 1994, ByRne 2002).

Para as vocalizações, foi utilizado um índice de intensidade, conforme o National American Amphibian Monitoring Population, sendo: (0) nenhum indivíduo da espécie vocalizando; (1) vocal izações esparsas, sem sobreposi ção e número de indivíduos estimável entre 1 e 10; (2) vocalizações se sobrepõe, mas ainda é possível individualizá-las e estimar o número de indivíduos (11-35 indivíduos); (3) formação de coro em que as vocal izações individuais são indistinguíveis e não se pode estimar o número de indivíduos (> 35) (WEIR 2001 apud CROUCH \& Paton 2002).

No período de estiagem, os registros foram realizados durante uma hora diária, entre 18:00 e 24:00 h. Na época chuvosa, o horário de início e término (turno) das vocal izações de cada espécie foi considerado a cada hora, a partir de 18:00 h até não haver mais registros de vocalizações. Dados abióticos

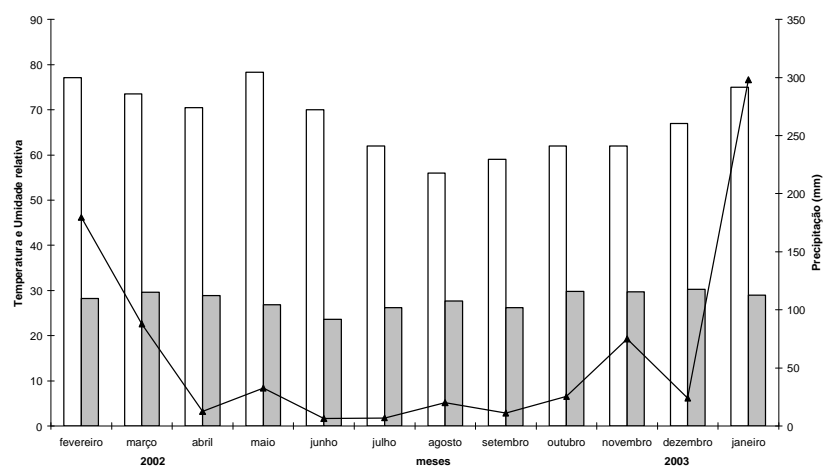

Figura 1. Médias da precipitação (linha), temperatura (barras cinzas) e umidade relativa do ar (barras brancas) em Corumbá, Mato Grosso do Sul, no período de fevereiro de 2002 a janeiro de 2003. Fonte: Ministério da Aeronáutica, Divisão Corumbá.

como temperatura, umidade relativa do ar, chuva e disponibilidade de água na lagoa foram registrados para a verificação da influência destes nas atividades de vocalizações das espécies. As médias mensais de temperatura, umidade relativa do ar e precipitação mensal foram fornecidas pela Divisão de Aeronáutica de Corumbá. A largura, comprimento e profundidade da lagoa foram medidas a cada mês.

Os micro-hábitats e substratos utilizados para as vocalizações, tais como tipo de vegetação e distância da água foram utilizados para a determinação da distribuição espacial dos anuros. A determinação dos modos reprodutivos foi classificada conforme Duellman \& TRUEB (1994), a partir da observação do tipo de desova, amplexo e outros fatores. A classificação e apresentação das espécies registradas seguiram Frost (2002).

\section{RESULTA DOS E DISCUSSÃ O}

\section{Riqueza específica}

Foram registradas16 espécies, distribuídas em quatro famílias e nove gêneros (Tab. I). A anurofauna é composta, predominantemente, pelas famílias Hylidae $(43,75 \%)$ e Leptodactylidae (37,5\%), fato comum na região Neotropical (DUELLMAN \& TRUEB 1994) e registrado para outras localidades do Brasil (Bertoluci \& Rodrigues 2002).

Entre todas as espécies, 75\% foram encontradas também em habitações humanas. Scinax acuminatus (Cope, 1862) foi encontrada apenas em habitações nas proximidades dos locais amostrados, não havendo registro de sua vocalização na mata ou em torno da lagoa temporária. Três espécies (Bufo schneideri Werner, 1894, Phrynohyas venulosa (Laurenti, 1768) e Scinax fuscovarius (A. Lutz, 1925)) vocalizaram fora do período de registro, não tendo sido considerada a densidade de indivíduos vocalizando.

\section{Distribuição espacial}

Das 16 espécies registradas, nove (56,25\%) são terrestres, seis $(37,5 \%)$ arborícolas e uma aquática $(6,25 \%)$. Os micro- 
Tabela I. Lista das famílias e espécies de anfíbios anuros, habito e microhabitats registrados em uma área urbana de Corumbá (MS), no período de fevereiro de 2002 a janeiro de 2003. (ARB) Arbustos, (ARV) árvores, (HAB) habitações humanas, (NA) nível da água, (SM) solo na margem do corpo d'água, (SS) solo seco, (VH) vegetação herbácea, (VA) valas e bueiros.

\begin{tabular}{|c|c|c|c|}
\hline Famílias & Espécies & Hábito & Microhabitat \\
\hline \multirow[t]{2}{*}{ Bufonidae } & Bufo granulosus & Terrestre & $\mathrm{SM}, \mathrm{HAB}$ \\
\hline & Bufo schneideri & Terrestre & $\mathrm{SM}, \mathrm{HAB}$ \\
\hline \multirow[t]{7}{*}{ Hylidae } & Hyla nana & Arborícola & $\mathrm{VH}$ \\
\hline & Phrynohyas venulosa & Arborícola & ARB, ARV, HAB \\
\hline & Scinax acuminatus & Arborícola & HAB \\
\hline & Scinax fuscovarius & Arborícola & SS, HAB \\
\hline & Scinax nasicus & Arborícola & $\mathrm{VH}, \mathrm{ARB}, \mathrm{HAB}$ \\
\hline & Phyllomedusa hypochondrialis & Arborícola & ARB \\
\hline & Pseudis paradoxa & Aquático & NA \\
\hline Microhylidae & Dermatonotus muelleri & Terrestre & NA, HAB \\
\hline \multirow[t]{6}{*}{ Leptodactylidae } & Leptodactylus chaquensis & Terrestre & $\mathrm{SM}, \mathrm{HAB}$ \\
\hline & Leptodactylus fuscus & Terrestre & SS, HAB \\
\hline & Leptodactylus labyrinthicus & Terrestre & $\mathrm{SM}, \mathrm{HAB}$ \\
\hline & Leptodactylus mystacinus & Terrestre & SS \\
\hline & Leptodactylus syphax & Terrestre & $H A B, V A$ \\
\hline & Physalaemus albonotatus & Terrestre & NA, HAB \\
\hline
\end{tabular}

habitats utilizados para a vocalização foram: a) valas de escoamento de água e bueiros, distante do corpo d'água: Leptodactylus syphax Bokermann, 1969; b) Flutuando ao nível da água: Pseudis paradoxa (Linnaeus, 1758), Physalaemus albonotatus e Dermatonotus muelleri (Boettger, 1885); c) Solo seco: Leptodactylus fuscus (Schneider, 1799), Leptodactylus mystacinus (Burmeister, 1861) e Scinax fuscovarius; d) Margem do corpo d'agua, sobre o solo encharcado: Leptodactylus chaquensis Cei, 1950 e Leptodactylus labyrinthicus (Spix, 1824); e) Margem do corpo d'água, com os membros submersos: Bufo granulosus Spix, 1824 eBufo schneideri; f) Sobre a vegetação herbácea marginal: Hyla nana Boulenger, 1889 e Scinax nasicus (Cope, 1862); g) Sobre a vegetação arbustiva marginal, menos de 1,5 m de altura: Scinax naiscus e Phyllomedusa hypochondrialis (Daudin, 1800); h) Sobre a vegetação arbustiva e arbórea, mais de 1,5m de altura: Phrynohyas venulosa.

Em estudo realizado em área de mata em Rio Claro (São Paulo), Toledo et al. (2003) encontraram seis espécies comuns ao presente estudo e utilizando os mesmos microhabitats: Bufo schneideri, Hyla nana, Scinax fuscovarius, Leptodactylus fuscus, L. labyrinthicus eL. mystacinus. Segundo HöDL (1977), a utilização de sítios de vocalização distintos tem grande importância no reconhecimento entre os indivíduos da mesma espécie, auxiliando no isolamento reprodutivo.

Foram encontrados quatro tipos de modos reprodutivos dentre as espécies (segundo Duellman \& Trueb 1994): Modo 1: ovos depositados diretamente na superfície da água. Presente nas espécies de Bufonidae (com a colocação dos ovos em cordões sobre a superfície) e nas espécies de Hylidae, com exceção de Phyllomedusa hypochondrialis. Modo 8: construção deninhos de espuma diretamente na água. Presente em Leptodactylus chaquensis, L. Iabyrinthicus e Physalaemus albonotatus (Steindachner, 1864). Modo 18: postura dos ovos na vegetação acima da água. Presente apenas em Phyllomedusa hypochondrialis. Modo 21: escavação de tocas e construção de ninhos de espuma em seu interior. Utilizado por Leptodactylus fuscus e L. mystacinus. Pertence a este mesmo modo a colocação do ninho de espuma em valas potenciais para escoamento de água, utilizado por Leptodactylus syphax.

Segundo Duellman \& Trueb (1994), a postura de ovos diretamente na superfície da água (modo 1 ) trata-se do meio mais primitivo e generalizado de reprodução entre os anuros e os modos 8 e 21 um tendência à terrestrialidade, sendo este último mais derivado. A tendência à oviposição no meio terrestre podem ser adaptações para evitar a dessecação e minimizar os efeitos de eventuais alterações no meio aquático (DueLLMAN \& TRUEB 1994) ou para reduzir os efeitos da predação sobre os ovos (MAgnusson \& HeRo 1991).

\section{Distribuição temporal}

O número de espécies vocal izando esteve correlacionado com a estação chuvosa (dezembro a março) $\left(r^{2}=0,806, F(1,10)\right.$ $=41,530 p=0,002, n=12$ ) (Fig. 2). Segundo Duellman \& Trueb (1994), a precipitação é o principal fator influenciando a ativi- 


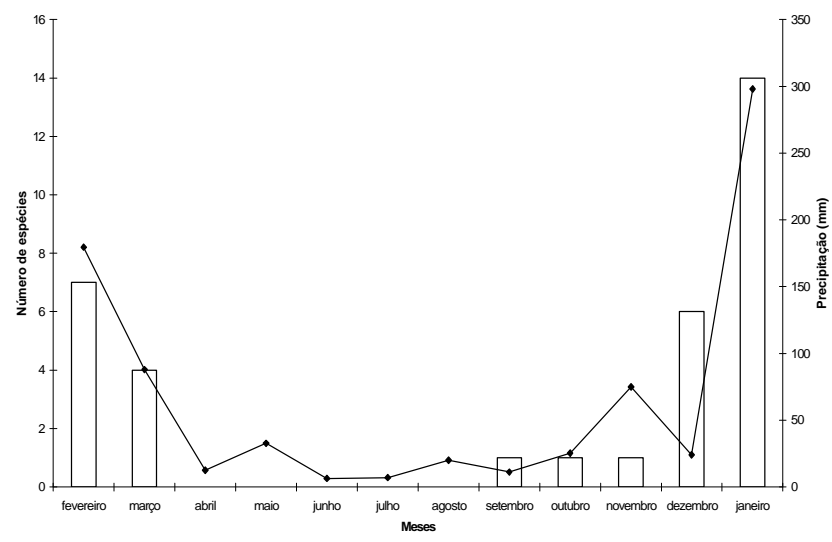

Figura 2. Relação entre a precipitação e o número de espécies vocalizando em área urbana de Corumbá, Mato Grosso do Sul, no período de fevereiro de 2002 a janeiro de 2003.

dade reprodutiva dos anuros nos trópicos. O número de espécies vocalizando não se relacionou com a média da temperatura do $\operatorname{ar}\left(r^{2}=0,13 F(1,10)=1,620 p=0,232 n=12\right)$ e a média da umidade relativa do $\operatorname{ar}\left(r^{2}=0,234 \mathrm{~F}(1,10)=3,055 \mathrm{p}=0,111 \mathrm{n}=\right.$ 12) mensais, queé compreensível uma vez que a flutuação dessas variáveis (média mensal) ao longo do ano foi baixa (Fig. 1).
A precipitação é responsável pela formação da lagoa temporária que no mês de janeiro atingiu a maior dimensão. A utilização de corpos d'água temporários para a reprodução entre os anuros pode ser devida à ausência de potenciais predadores aquáticos e maior disponibilidade de microambientes, como gramíneas e arbustos (AzEvedo-RAmos et al. 1999).

O número de espécies em atividade de vocalização variou ao longo do ano, com poucas espécies vocalizando por longos períodos. Seis espécies (Bufo granulosus, B. schneideri, Dermatonotus muelleri, Leptodactylus labyrinthicus, Scinax fuscovarius e Phrynohyas venulosa) apresentaram padrão explosivo de reprodução, associado às chuvas mais fortes (Tab. II).

Com relação à intensidade de vocalizações, Scinax nasicus e Physalaemus albonotatus foram as espécies com maior número de indivíduos (>35) vocalizando em janeiro. O período de vocalização de Leptodactylus fuscus foi o mais longo (setembro a fevereiro); entretanto, os machos em setembro e outubro não se encontravam próximos às tocas, local de deposição dos ninhos de espuma, o que pode indicar que a reprodução só ocorra de dezembro a fevereiro. Corroborando essa observação, ScotT \& WoodWARD (1994) afirmaram que a vocalização em anuros não significa exatamente reprodução, tornando-se necessária a observação de outros fatores, como amplexo, ovos ou larvas, para a determinação do período reprodutivo.

No entanto, em dezembro, os machos dessa espécie fo-

Tabela II. Número de espécies, modo reprodutivo, densidade de indivíduos vocalizando, total de espécies vocalizando e profundidade da coluna d'água em uma área urbana de Corumbá (MS), no período de fevereiro de 2002 a janeiro de 2003. As espécies que vocalizaram fora do período de coleta estão assinaladas com "x". (1) 1 a 10 indivíduos, (2) 11 a 35 indivíduos, (3) mais de 35 indivíduos.

\begin{tabular}{|c|c|c|c|c|c|c|c|c|c|c|c|c|c|}
\hline \multirow{2}{*}{ Espécies } & \multirow{2}{*}{ Modo reprodutivo } & \multicolumn{12}{|c|}{ Meses } \\
\hline & & Fev & Mar & $A b r$ & Mai & Jun & Jul & Ago & Set & Out & Nov & Dez & Jan \\
\hline Bufo granulosus & 1 & & & & & & & & & & & & 1 \\
\hline Bufo schneideri & 1 & & & & & & & & & & & & $x$ \\
\hline Hyla nana & 1 & 1 & 2 & & & & & & & & & & 1 \\
\hline Phrynohyas venulosa & 1 & & & & & & & & & & & & $x$ \\
\hline Scinax fuscovarius & 1 & & & & & & & & & & & & $x$ \\
\hline Scinax nasicus & 1 & 2 & & & & & & & & & & & 3 \\
\hline Phyllomedusa hypochondrialis & 18 & 1 & 1 & & & & & & & & & 1 & 1 \\
\hline Pseudis paradoxa & 1 & 1 & 1 & & & & & & & & & & 1 \\
\hline Dermatonotus muelleri & 1 & & & & & & & & & & & & 1 \\
\hline Leptodactylus chaquensis & 8 & & & & & & & & & & & 1 & 1 \\
\hline Leptodactylus fuscus & 21 & 1 & & & & & & & 1 & 1 & 1 & 2 & 1 \\
\hline Leptodactylus labyrinthicus & 8 & & & & & & & & & & & & 1 \\
\hline Leptodactylus mystacinus & 21 & & & & & & & & & & & 1 & \\
\hline Leptodactylus syphax & 8 & 1 & & & & & & & & & & 1 & 1 \\
\hline Physalaemus albonotatus & 8 & 2 & 1 & & & & & & & & & 1 & 3 \\
\hline Profundidade da água (mm) & & 200 & 110 & 0 & 0 & 0 & 0 & 0 & 0 & 0 & 0 & 85 & 226 \\
\hline Total de espécies vocalizando & & 7 & 4 & 0 & 0 & 0 & 0 & 0 & 1 & 1 & 0 & 6 & 14 \\
\hline
\end{tabular}

Revista Brasileira de Zoologia 21(4): 887-892, dezembro 2004 
Tabela III. Turno de vocalizações (17:00 às 06:00 h) das espécies de anuros em uma área urbana de Corumbá, Mato Grosso do Sul, de fevereiro de 2002 a janeiro de 2003.

\begin{tabular}{|c|c|c|c|c|c|c|c|c|c|c|c|c|c|}
\hline Espécies & $\begin{array}{l}17: 00 \\
18: 00\end{array}$ & $\begin{array}{l}18: 01 \\
19: 00\end{array}$ & $\begin{array}{l}19: 01 \\
20: 00\end{array}$ & $\begin{array}{l}20: 01 \\
21: 00 \\
\end{array}$ & $\begin{array}{c}21: 01 \\
2: 00 \\
\end{array}$ & $\begin{array}{l}22: 01 \\
23: 00 \\
\end{array}$ & $\begin{array}{l}23: 01 \\
00: 00\end{array}$ & $\begin{array}{l}00: 01 \\
01: 00\end{array}$ & $\begin{array}{l}01: 01 \\
02: 00\end{array}$ & $\begin{array}{l}02: 01 \\
03: 00\end{array}$ & $\begin{array}{l}03: 01 \\
04: 00\end{array}$ & $\begin{array}{l}04: 01 \\
05: 00\end{array}$ & $\begin{array}{l}05: 01 \\
06: 00\end{array}$ \\
\hline Bufo granulosus & & & & $x$ & $x$ & $x$ & & & & & & & \\
\hline Hyla nana & & & $x$ & $x$ & $x$ & $x$ & $x$ & & & & & & \\
\hline Phrynohyas venulosa & & & $x$ & $x$ & $x$ & $x$ & $x$ & $x$ & $x$ & & & & \\
\hline Scinax nasicus & $x$ & $x$ & $x$ & $x$ & $x$ & $x$ & $x$ & $x$ & $x$ & $x$ & $x$ & $x$ & \\
\hline Phyllomedusa hypocondrialis & & & $x$ & $x$ & $x$ & $x$ & $x$ & $x$ & $x$ & $x$ & $x$ & $x$ & \\
\hline Pseudis paradoxa & $x$ & $x$ & $x$ & $x$ & $x$ & $x$ & $x$ & & & & & & \\
\hline Dermatonotus muelleri & & & & $x$ & $x$ & $x$ & $x$ & $x$ & $x$ & $x$ & $x$ & & \\
\hline Leptodactylus chaquensis & $x$ & $x$ & $x$ & $x$ & $x$ & $x$ & & & & & & & \\
\hline Leptodactylus fuscus & & $x$ & $x$ & $x$ & $x$ & $x$ & $x$ & $x$ & $x$ & $x$ & $x$ & & \\
\hline Leptodactylus labyrinthicus & & & & & $x$ & $x$ & $x$ & $x$ & $x$ & & & & \\
\hline Leptodactylus mystacinus & & & $x$ & $x$ & & & & & & & & & \\
\hline Leptodactylus syphax & & $x$ & $x$ & $x$ & $x$ & $x$ & $x$ & & & & & & \\
\hline Physalaemus albonotatus & $x$ & $x$ & $x$ & $x$ & $x$ & $x$ & $x$ & $x$ & $x$ & $x$ & $x$ & $x$ & $x$ \\
\hline Total de espécies vocalizando & 4 & 6 & 10 & 12 & 12 & 12 & 10 & 7 & 7 & 5 & 5 & 3 & 1 \\
\hline
\end{tabular}

ram avistados vocalizando próximos às suas tocas e a densidade de indivíduos nesse mês foi maior que em janeiro e fevereiro, o que pode significar maior esforço reprodutivo no período de menor riqueza de anuros, onde a competição pelo espaço acústico é menor.

A concentração das vocalizações (maior número de espécies vocal izando) ocorreu nos horários entre 19:00 e 23:00h (Tab. III). CARDOSO \& MARTINS (1987) constataram tendência à concentração da atividade de vocalização entre 19:00 e 22:00 h no Sudeste do Brasil, inferindo que o encerramento das atividades na segunda metade da noite possa estar relacionado à economia de energia, necessidade alimentar e diminuição da temperatura.

Mesmo nessa pequena porção da área urbana de Corumbá, foi registrado um número relativamente alto $(45,7$ \%) de espécies de anuros, quando comparado com aquelas encontradas no Pantanal (ver PCBAP 1997), levando a acreditar que mesmo em se tratando de área impactada e de mata secundária, o local oferece condições para a reprodução. Estudos com enfoque em abundância, épocas e horários adequados para a reprodução das espécies de anuros podem favorecer trabal hos futuros de monitoramento e manejo das populações, a fim de se evitar a eliminação desses animais do local.

\section{AGRADECIMENTOS}

À Companhia de Cimento Itaú pelo livre acesso à área, ao Ministério da Aeronáutica, Divisão Corumbá, por disponibilizar os dados climáticos. A ChristineStrussmann, José A. Langone, Masao Uetanabaro, Ulisses Caramaschi e um con- sultor anônimo pela leitura do manuscrito e valiosas sugestões.

\section{REFERÊNCIAS BIBLIO GRÁ FICAS}

Azevedo-Ramos, C.; W.E. Magnusson \& P. Bayliss. 1999. Predation as the key factor structuring tadpole assemblages in a savanna área in Central Amazônia. Copeia, Lawrence, 1999 (1): 22-33.

Bertolucl, J. \& M.T. Rodrigues. 2002. Utilização de habitats reprodutivos e micro-habitats de vocal ização em uma taxocenose de anuros (amphibia) da M ata Atlântica do sudeste do Brasil. Papéis Avulsos de Zoologia, São Paulo, 42 (11): 287297.

BYRNE, P.G. 2002. Climatic correlates of breeding, simultaneous polyandry and potential for sperm competition in the frog Crinia georgiana. Journal of Herpetology, Lawrence, 36 (1): 125-129.

CARdoso, A.J. \& J.E. Martins. 1987. Diversidade de anuros durante o turno de vocalizações, em comunidade neotropical. Papéis Avulsos de Zoologia, São Paulo, 36 (23): 279-285.

CARR, L.W. \& L. FAHRIG. 2001. Effect of road traffic on two amphibian species of differing vagility. Conservation Biology, Malden, 15 (4): 1071-1078.

Crouch III, W.B. \& P.W.C. Paton. 2002. Assessing the use of call surveys to monitor breeding anurans in Rhode Island. Journal of Herpetology, Lawrence, 36 (2): 185-192.

Duellman, W.E. \& L. Trueb. 1994. Biology of Amphibians. Baltimore, The Johns Hopkins University Press, 670p.

Ferrier, U. 2002. Monitorizacíon de anfibios. Reptilia, Barcelona, 33: 63-65.

Revista Brasileira de Zoologia 21(4): 887-892, dezembro 2004 
Frost, D.R. 2002. Amphibian Species of the World: an online reference. Versão 2.21 (15 July 2002). Disponível em http:/ /research.amnh.org/herpetology/amphibia/index.html.

HodgkISON, S. \& J. Hero. 2001. Daily behavior and microhabitat use of the waterfall frog, Littoria nannotis in Tully Gorge, Eastern Australia. Journal of Herpetology, Lawrence, 35 (1): 116-120.

HöDL, W. 1977. Call Differences and Calling Site Segregation in Anuran Species from Central Amazonian Floating Meadows. Oecologia, Heidel berg, 28: 351-363.

JANSEN, K.P.; A.P. SUMmers \& P.R. Delis. 2001. Spadefoot toads (Scaphiopus holbrookii) in a urban landscape: effects of non natural substrates on burrowing in adults and juveniles. Journal of Herpetology, Lawrence, 35 (1): 141-145.

Koenig, J.; R. Shine \& G. Shea. 2002. The dangers of life in city: patterns of activity, injury, and mortality in suburban lizards (Tiliqua scincoides). Journal of Herpetology, Lawrence, 36 (1): 62-68.

Magnusson, W.E. \& J. Hero. 1991. Predation and the evolution of complex oviposition behaviour in Amazon rainforest frogs. Oecologia, Heidelberg, 86: 310-318.

MARSH, D.M. 2001. Fluctuations in amphibian populations: a meta-analysis. Biological Conservation, Davis, (101): 327335.

Mazerolle, M.J. 2001. Amphibian activity, movement patterns and body size in fragmented peat bogs. Journal of Herpetology, Lawrence, 35 (1): 13-20.

Papp, M.G. \& C.O.G. Papp. 2000. Decline in a population of the treefrog Phyllodytes luteolus after fire. Herpetological Review, Lawrence, 31 (2): 93-95.

PcBAp (Plano de Conservação da Bacia do Alto Paraguai). 1997. Programa Nacional do Meio Ambiente. Braślia, PNMA, vol. 3.

Pechmann, J.H.K.; D.E. ScotT; R.D. Semlitsch; J.P. Caldwell; L.J. VITT \& W. GIBBONS. 1991.Declining amphibians populations: the problem of separating human impacts from natural fluctuations. Science, Washington, 253: 825-940.

ScotT JR., N. \& B.D. W OodWARD. 1994. Surveys at breeding sites, p.118-125. In: HeYER, W.R.; M.A. DONNELlY; R.W. MCDIARMID; L.C. HAYEK \& M.S. FOSTER (Eds). Measuring and Monitoring Biological Diversity - Standard Methods for Amphibians. Washington, Smithsonian Institution Press, 364p.

Seebacher, F. \& R.A. Alford. 1999. Movement and microhabitat use of a terrestrial amphibian (Bufo marinus) on a tropical island: seasonal variation and environmental correlates. Journal of Herpetology, Lawrence, 33 (2): 208-214.

SoRIANO, B.M.A. 1997. Caracterização climática de CorumbáMS. Corumbá, EMBRAPA-CPAP, 25p.

SUMmeRS, K. 2002. Forests for the frogs, frogs for the forests. Herpetological Review, Lawrence, 33 (1): 16-18.

Toledo, L.F.; J. ZINA \& C.F.B. HADDAD. 2003. Distribuição Espacial e Temporal de uma Comunidade de Anfíbios Anuros do Município de Rio Claro, São Paulo, Brasil. Holos Environment, Rio Claro, 3 (2): 136-149.

WILSON, J.J. \& T.J. M ARET. 2002. A comparison of two methods for estimating the abundance of amphibians in aquatic habitats. Herpetological Review, Lawrence, 33 (2): 108-110.

Recebido em 09.III.2004; aceito em 10.XI.2004.

Revista Brasileira de Zoologia 21(4): 887-892, dezembro 2004 